\title{
Brief Report: The Use of WAIS-III in Adults with HFA and Asperger Syndrome
}

\author{
Antoinette A. Spek • Evert M. Scholte • \\ Ina A. van Berckelaer-Onnes
}

Published online: 19 September 2007

(C) Springer Science+Business Media, LLC 2007

\begin{abstract}
The WAIS III was administered to 16 adults with high functioning autism (HFA) and 27 adults with Asperger syndrome. Differences between Verbal Intelligence (VIQ) and Performance Intelligence (PIQ) were not found. Processing Speed problems in people with HFA appeared. At the subtest level, the Asperger syndrome group performed weak on Digit Span. Comprehension and Block Design were relative strengths. In the HFA group, performance on Digit-Symbol Coding and Symbol Search was relatively poor. Strengths were found on Information and Matrix Reasoning. The results suggest that the VIQPIQ difference cannot distinguish between HFA and Asperger syndrome. WAIS III Factor Scale and Subtest patterning provides a more valid indicator.
\end{abstract}

Keywords High functioning autism .

Asperger syndrome $\cdot$ Intelligence $\cdot$ WAIS-III ·

Processing speed

\section{Introduction}

Over the past few years, interest in HFA and Asperger syndrome in adults with normal intelligence has increased markedly. However, not much is known about the cognitive profiles of these groups. Only a few studies exist about adults who function relatively well in society and have

A. A. Spek $(\bowtie)$

GGZ Eindhoven, Boschdijk 771, Postvak 1418, Eindhoven 5626

AB, The Netherlands

e-mail: aa.spek@ggze.nl

E. M. Scholte · I. A. van Berckelaer-Onnes

Department of Clinical Child and Adolescent Studies, Leiden

University, Leiden, The Netherlands been diagnosed late in life (Howlin 2004; Vermeulen 2002). The present study aims to assess the cognitive profiles of this relatively well-functioning subgroup by means of the Wechsler Intelligence Scale III (WAIS III, Wechsler 1997).

In WAIS III, the intelligence pattern is described at three levels: The first level contains Performance Intelligence and Verbal Intelligence. The second level consists of the four factor scales: Verbal Comprehension, Perceptual Organization, Freedom from Distractibility and Processing Speed. The third level contains the subtests. The following paragraphs summarizes research on the intelligence profiles of adults with the autistic disorder or Asperger syndrome on the basis of these three levels.

The Performance IQ (PIQ)-Verbal IQ (VIQ) dichotomy has been incorrectly used for years to underpin the diagnosis of autistic disorder or Asperger syndrome. It is questionable whether the two constructs should even be applied in general, because research did not support the construct validity of the VIQ-PIQ dichotomy (Taub 2001).

For adults with HFA, studies on WAIS-R have yielded contradictory results (Minshew et al. 1992; Siegel et al. 1996; Vermeulen 2002), which may reflect the validity problems of the VIQ-PIQ dichotomy (Arnau and Thompson 2000; Taub 2001).

The factor scale level is of great importance in assessing cognitive abilities because factor analytic studies indicate that the factor scales give the best estimates of the four factors underlying intelligence (Arnau and Thompson 2000; Ryan and Paolo 2001).

No studies have been performed on WAIS-III profiles for adults with HFA or Asperger syndrome as far as we know. Therefore no information is available on the results of factor scales in these groups. This leads to the conclusion that the most important factors of the intelligence 
pattern for adults with HFA or Asperger syndrome are still unknown.

On subtest level, some studies on WAIS or WAIS-R found low Comprehension versus high Block Design scores (Goldstein et al. 2001; Rumsey and Hamburger 1988). A relatively high variability between the subtests scores in adults with HFA has also been reported (Siegel et al. 1996).

In summary, research shows that among adults with HFA or Asperger syndrome, results of VIQ-PIQ differences vary and may be influenced by the validity problems of the VIQ-PIQ dichotomy. The factor scale scores and the subtest patterns provide a better representation of the intelligence pattern.

\section{Aims of the Present Study}

The present study aims to acquire insight into the WAIS III profiles of normal intelligent adults with HFA and Asperger syndrome. Profiles in the total group and differences between the two diagnostic groups will be examined.

\section{Methods}

\section{Procedure}

All participants were recruited from the GGZ (Mental Health Center) Eindhoven and Oost-Brabant. The participants met the criteria for Asperger syndrome or HFA. Participants with relevant neurodevelopmental conditions and genetic conditions were excluded, as were institutionalized patients and patients with a Full Scale IQ below 80 .

\section{Subjects}

The mean Full Scale IQ of the participants was 110.16, individual scores varied between 83 and 145 (see Table 1).

All individuals ranged in age from 18 to 60 years. The mean age was 41.93. Of all participants, 25 finished higher education and 30 individuals had work. 23 participants lived together with a partner. The relatively large number of participants who had a relationship, worked and were well educated emphasizes the relatively high level of functioning in this group.

\section{Assessment of Disorder}

Hetero-anamnestic information was gathered using the Dutch version of the Autistic Disorder Diagnostic
Table 1 Characteristics of participants

\begin{tabular}{llll}
\hline IQ and age & M & SD & Range \\
$\quad$ Full scale IQ & 110.16 & 16.05 & $83-145$ \\
$\quad$ Mean age & 41.93 & 10.67 & $20-60$ \\
Diagnosis & $n$ & $\%$ & \\
$\quad$ Autistic disorder & 16 & 37.2 & \\
$\quad$ Asperger syndrome & 27 & 62.8 & \\
Gender & & & \\
$\quad$ Male & 39 & 90.7 & \\
Female & 4 & 9.3 & \\
Education & & & \\
$\quad$ Lower/middle education & 18 & 41.9 & \\
$\quad$ Higher education & 25 & 58.1 & \\
Employment status & & & \\
Employed or retired & 30 & 69.8 & \\
Studying & 1 & 2.3 & \\
$\quad$ Unemployed & 12 & 27.9 & \\
Current living circumstances & & & \\
Lives with partner & 23 & 53.5 & \\
Lives independently & 12 & 27.8 & \\
Sheltered living & 2 & 4.7 & \\
Lives with parents & 6 & 14.0 & \\
\hline
\end{tabular}

Interview, revised version (ADI-R, Lord et al. 1994), administered by psychologists who were officially trained in the administration and scoring of the instrument. To gather anamnestic information, a semi-structured interview was used to assess presence of the DSM-IV criteria of HFA and Asperger syndrome (APA 1994). Because of the controversial nature of the DSM-IV criteria (Ghaziuddin et al. 1992; Mayes et al. 2001), additional questions were used to differentiate between HFA and Asperger syndrome, based on the diagnostic criteria of Gillberg and Gillberg (1989) and ICD-10 (WHO 1993).

\section{Assessment of Intelligence}

The intelligence profile was assessed using the Dutch translation of the WAIS III (Wechsler 1997). The WAISIII has excellent psychometric properties (Sattler and Ryan 1999) and has been validated for the Dutch population (Wechsler 1997).

\section{Results}

Analyses were done at the three WAIS-III levels: VIQ versus PIQ, the four factor scales and all subtests. Preliminary analysis included checks for normality, linearity, influential data points and assumptions of repeated 
Table 2 VIQ and PIQ differences in the total group and in diagnostic groups

\begin{tabular}{llllllll}
\hline & VIQ & & & & PIQ & \multicolumn{3}{c}{ Mean difference $n$} \\
\cline { 2 - 3 } & $\mathrm{M}$ & $\mathrm{SD}$ & & $\mathrm{M}$ & $\mathrm{SD}$ & & \\
\hline Total group & 110.30 & 13.83 & & 108.42 & 18.21 & 1.88 & 43 \\
Asperger & 111.41 & 13.57 & & 112.52 & 17.28 & 1.11 & 27 \\
Autistic disorder & 108.44 & 14.49 & & 101.50 & 18.13 & 6.94 & 16 \\
\hline
\end{tabular}

measures. No serious deviations were found. T-tests showed that both diagnosis groups were comparable in education and work status, as well as in gender distribution.

\section{Differences Between WAIS III VIQ and PIQ}

Differences between VIQ and PIQ for all participants and both diagnostic groups were analyzed by means of paired $t$ tests. No statistically significant effects were found for any of the investigated groups (see Table 2).

\section{Differences Between Factor Scale Scores}

Factor Scale profiles were studied within the total group and between the two diagnostic subgroups by means of repeated measures analysis of variance. Mauchly's test indicated that the assumption of sphericity was not met. Therefore the degrees of freedom were corrected using the Huynh-Feldt correction $(\varepsilon=.89)$. Post-hoc comparisons using the Sidak adjustment for multiple comparisons showed that the main effect of the WAIS III Factor Scale was statistically significant $(F(2.7,109.7)=7.0$, $p<0.001)$. An interaction effect of differences in Factor Scale mean by diagnostic group was also found $(F(2.7$, $109.7)=2.7, p=0.05$ ). To find out which differences in WAIS III Factor Scale means added to the significant main effect, post hoc pairwise comparisons were done. This showed that the main effect in the total group can be attributed to Processing Speed being significantly lower than Verbal Comprehension $(p<.01)$ and Perceptual Organization $(p<.005)$.

Post hoc pairwise comparisons were done for the two diagnostic groups to analyse the 'within group' effect. In the Asperger group, no significant differences in Factor Scale mean scores were found. The HFA group however, showed a significant lower Processing Speed compared to Verbal Comprehension $(p<.01)$, Perceptual Organization $(p<.01)$ and Freedom from Distractibility $(p<.05)$ (see Table 3).
Table 3 Factor scale scores for the total group and the diagnostic groups

\begin{tabular}{llll}
\hline Factor scale & $\mathrm{M}$ & $\mathrm{SD}$ & $\mathrm{N}$ \\
\hline Verbal comprehension & & & \\
Autistic disorder & $107.5^{*}$ & 12.1 & 16 \\
Asperger syndrome & 110.8 & 11.9 & 27 \\
Total & $109.6^{*}$ & 12.0 & 43 \\
Perceptual organization & & & \\
Autistic disorder & $105.0^{*}$ & 18.7 & 16 \\
Asperger syndrome & 111.8 & 13.0 & 27 \\
Total & $109.3^{*}$ & 15.5 & 43 \\
Freedom from distractibility & & & \\
Autistic disorder & $105.1^{*}$ & 18.2 & 16 \\
Asperger syndrome & 107.2 & 15.4 & 27 \\
Total & 106.4 & 16.3 & 43 \\
Processing speed & & & \\
Autistic disorder & $91.8^{*}$ & 17.4 & 16 \\
Asperger syndrome & 106.5 & 19.4 & 27 \\
Total & $101.0^{*}$ & 19.8 & 43 \\
\hline
\end{tabular}

$* p<.05$.

\section{Differences Between WAIS III Subtest Scores}

The Subtest profiles were explored within the total group and between the two diagnostic subgroups by means of a repeated measures analysis of variance. The assumption of sphericity was not met. Therefore the degrees of freedom were corrected using the Huynh-Feldt correction $(\varepsilon=.82)$. Post-hoc comparisons were performed using the Sidak adjustment for multiple comparisons. The results (see Table 4) showed a significant main effect of the type of Subtest $(F(10.7,438.7)=4.8, p<0.001)$.

Table 4 Mean standardized subtest scores for the total group

\begin{tabular}{llll}
\hline Subtest scores & $\mathrm{M}$ & $\mathrm{SD}$ & $\mathrm{n}$ \\
\hline Vocabulary & $11.63^{*}$ & 2.564 & 43 \\
Similarities & 11.42 & 2.490 & 43 \\
Arithmetic & $11.77^{*}$ & 3.046 & 43 \\
Digit span & 10.72 & 3.268 & 43 \\
Information & $12.42^{*}$ & 2.779 & 43 \\
Comprehension & $12.53^{*}$ & 2.772 & 43 \\
Letter-number sequencing & 10.98 & 2.956 & 43 \\
Picture completion & 10.88 & 3.253 & 43 \\
Digit-Symbol Coding & $9.81^{*}$ & 3.438 & 43 \\
Block design & $12.02^{*}$ & 3.562 & 43 \\
Matrix reasoning & $11.98^{*}$ & 2.454 & 43 \\
Picture arrangement & 11.53 & 3.731 & 43 \\
Symbol search & $10.37^{*}$ & 3.970 & 43 \\
Object assembly & 11.16 & 3.086 & 43 \\
\hline
\end{tabular}

$* p<.05$. 
Table 5 Mean standardized subtest scores for the autistic disorder group

\begin{tabular}{lccc}
\hline Subtest Scores & M & SD & N \\
\hline Vocabulary & 11.31 & 2.496 & 16 \\
Similarities & 10.94 & 1.769 & 16 \\
Arithmetic & 11.44 & 3.705 & 16 \\
Digit span & 11.31 & 3.400 & 16 \\
Information & $12.13^{*}$ & 3.284 & 16 \\
Comprehension & 11.75 & 2.176 & 16 \\
Letter-number sequencing & 10.25 & 3.152 & 16 \\
Picture completion & 10.81 & 4.070 & 16 \\
Digit-Symbol Coding & $8.38^{*}$ & 3.030 & 16 \\
Block design & 10.56 & 3.444 & 16 \\
Matrix reasoning & $11.44^{*}$ & 2.828 & 16 \\
Picture arrangement & 10.19 & 3.674 & 16 \\
Symbol search & $8.44^{*}$ & 3.483 & 16 \\
Object assembly & 9.88 & 3.324 & 16 \\
\hline
\end{tabular}

$* p<.05$.

An interaction effect of Subtest by diagnosis was also found $(F(10.7,438.7)=2.1, p<0.05)$, indicating that the patterning of the WAIS III subtest mean scores for the two diagnostic groups differs. Table 5 and 6 show the mean Subtest scores and standard deviations for the HFA group and the Asperger syndrome group.

Post hoc pair wise comparisons showed that the main effect in the total group can be attributed to the fact that Digit-Symbol Coding was significantly lower than Vocabulary $(p<.05)$, Arithmetic $(p<.05)$, Information $(p<.005)$, Comprehension $(p<.005)$, Block Design $(p<.05)$ and Matrix Reasoning $(p<.005)$. Furthermore, Symbol Search was lower than Information $(p<.05)$ and Comprehension $(p<.05)$.

Post hoc pair-wise comparisons were also performed for the two diagnostic groups to analyze the 'within group' effect. The two groups showed significant differences in Subtest scores. In the Asperger syndrome group, Digit Span was lower than Comprehension $(p=.005)$ and Block Design $(p<.05)$.

In the HFA group performance was significantly higher in Information compared to Digit-symbol Coding $(p<.05)$ and Symbol Search $(p<.05)$. Furthermore, Digit-Symbol Coding was lower than Matrix Reasoning $(p<.05)$.

\section{Discussion}

\section{WAIS VIQ Versus PIQ}

No significant differences were found between VIQ and PIQ in the total group nor in the two diagnostic subgroups.
Table 6 Mean standardized subtest scores for the Asperger syndrome group

\begin{tabular}{llll}
\hline Subtest scores & M & SD & $n$ \\
\hline Vocabulary & 11.81 & 2.632 & 27 \\
Similarities & 11.70 & 2.826 & 27 \\
Arithmetic & 11.96 & 2.638 & 27 \\
Digit Span & $10.37 *$ & 3.200 & 27 \\
Information & 12.59 & 2.485 & 27 \\
Comprehension & $13.00^{*}$ & 3.013 & 27 \\
Letter-number sequencing & 11.41 & 2.805 & 27 \\
Picture completion & 10.93 & 2.745 & 27 \\
Digit-Symbol Coding & 10.67 & 3.431 & 27 \\
Block design & $12.89 *$ & 3.401 & 27 \\
Matrix reasoning & 12.30 & 2.198 & 27 \\
Picture arrangement & 12.33 & 3.595 & 27 \\
Symbol search & 11.52 & 3.847 & 27 \\
Object assembly & 11.93 & 2.716 & 27 \\
\hline
\end{tabular}

$* p<.05$.

The results are in line with factor analytic studies showing that the VIQ-PIQ dichotomy is not valid for general populations (Arnau and Thompson 2000; Taub 2001).

\section{WAIS III Factor Scale Level}

The Asperger syndrome group was characterized by a flat Factor Scale profile in the Asperger syndrome group, while the HFA group performed significant low in Processing Speed. A low Processing Speed indicates problems in speed of processing visual information (Wechsler 1997). Adults with HFA apparently need more time than other people to process and integrate visual information and to act on this information.

The Processing Speed performance of the HFA group might be influenced by problems with top-down processing and ignoring irrelevant details, which are characteristic of people with HFA (Happé 2005; Shah and Frith 1993). In order to maintain an overview of what they are doing, they work slowly.

\section{WAIS III Subtest Level}

Analyses showed different Subtest patterns in the HFA and the Asperger syndrome groups. The HFA group performed significantly low in Digit-Symbol Coding and Symbol Search. These two subtests together form the Processing Speed Factor. The low scores for these subtests represent the problems in speed of processing visual information as described in the preceding paragraph. 
The HFA group showed significantly high performance in Information and Matrix Reasoning. High scores for Information are in line with the fact that people with autism usually acquire much factual knowledge (Happé 1999).

Matrix Reasoning taps nonverbal perceptual reasoning. Matrix Reasoning is the only Perceptual Organization subtest without a time limit and is possibly not influenced by low Processing Speed performance scores. The good performance of the HFA group can probably be attributed to their visual-spatial strengths (Lincoln et al. 1995; Tsatsanis 2005) and to the absence of a time limit for this subtest.

In the Asperger group, scores for Digit Span were relatively low. Digit Span taps working memory capabilities (Wechsler 1997), which can been defined as 'the ability to hold in mind past states of the environment and past actions while currently performing an action' (Russell 1997). People with autism or Asperger syndrome tend to store information in details instead of using strategies, which often leads to problems in retaining information (Happé 2005; Minshew et al. 1992; Tsatsanis 2005). Low Digit Span scores in the Asperger group may reflect problems in applying strategies to retain information.

The Asperger syndrome group performed significantly well on Comprehension. High scores on Comprehension in this group seem to contradict former research results (Klin et al. 2005; Mayes and Calhoun 2003; Siegel et al. 1996). However, people with Asperger syndrome often try to function in society by analyzing social situations at a cognitive level, which has been described as using an 'explicit theory of mind' (Frith and Happé 1999). A extremely well developed explicit theory of mind may have caused the Asperger syndrome group to have such high scores on Comprehension.

The Asperger Syndrome group also performed significantly well on Block Design. Strengths in Block Design have often been reported in studies of people with HFA or Asperger syndrome (Happé 2005; Shah and Frith 1993). This has been attributed to strengths in processing unconnected stimuli outside a meaningful context, which go together with the central coherence problems seen in people with autistic impairment (Shah and Frith 1993).

\section{Conclusions}

The present study found people with Asperger syndrome to differ significantly from people with HFA in WAIS III Factor Scale profiles and WAIS III Subtest patterning. In people with HFA Processing Speed problems were found. Further, the HFA and Asperger syndrome group showed different subtest patterns. The present study supports the idea that HFA and Asperger syndrome can be differentiated empirically at the level of intellectual functioning. This lends support to the hypothesis that HFA and the Asperger syndrome are two separate disorders.

Acknowledgements Present article was submitted as part of the first author's PhD Degree at the University of Leiden. The second and third authors are members of the Department of Clinical Child and Adolescent studies at Leiden University. The article was written while the first author was employed by the Mental Health Center Eindhoven (GGZ Eindhoven). Special thanks go to colleagues in Eindhoven and Oss who assisted in the study. We are grateful to all of the adults who participated in the present study.

\section{References}

American Psychiatric Association. (1994). Diagnostische criteria van de DSM-IV. Lisse: Swets \& Zeitlinger.

Arnou, R.C., \& Thompson, B. (2000). Second order confirmatory factor analysis of the WAIS-III. Assessment, 7(3), 237-246.

Frith, U., \& Happé, F. (1999). Theory of mind and self-consciousness: What is it like to be autistic? Mind and Language, 14(1), 1-22.

Ghaziuddin, M., Tsai, L. Y., \& ghaziuddin, N. (1992). A comparison of the diagnostic criteria for Asperger syndrome. Journal of Autism and Developmental Syndromes, 22, 634-639.

Gillberg, C., \& Gillberg, C. (1989). Asperger syndrome-some epidemiological considerations: A research note. Journal of Child Psychology and Psychiatry, 30(4), 631-638.

Goldstein, G., Beers, S. R., Siegel, D. J., \& Minshew, N. J. (2001). A comparison of WAIS-R profiles in adults with high-functioning autism or differing subtypes of learning disability. Applied Neuropsychology, 8(3), 148-154.

Happé, F. G. E. (1999). Autism cognitive deficit or cognitive style? Trends in Cognitive Sciences, 6(3), 216-222.

Happé, F. G. E. (2005). The weak central coherence account of autism. In F. R. Volkmar, P. Rhea, A. Klin, \& D. Cohen (Eds.), Handbook of autism and pervasive developmental disorders. New Jersey: John Wiley \& Sons. 772-798.

Howlin, P., Goode, S., Hutton, J., \& Rutter, M. (2004). Adult outcome for children with autism. Journal of Child Psychology and Psychiatry, 45(2), 212-229.

Klin, A., Saulnier, C., Tsatsanis, K., \& Volkmar, F. (2005). Clinical evaluation in autism spectrum disorders: Psychological assessment within a transdisciplinary framework. In F. R. Volkmar, P. Rhea, A. Klin, \& D. Cohen (Eds.), Handbook of autism and pervasive developmental disorders. New Jersey: John Wiley \& Sons. 772-798.

Lincoln, A. J., Allen, M. H., \& Kilman, A. (1995). The assessment and interpretation of intellectual abilities in people with autism. In E. Schopler, \& G. B. Mesibov (Eds.), Learning and cognition in autism. New York: Plenum Press. 89-117.

Lord, C., Rutter, M., \& LeCouteur, A. (1994). Autism diagnostic interview-revised: A revised version of a diagnostic interview for caregivers of individuals with possible pervasive developmental disorders. Journal of Autism and Developmental Disorders, 24, 659-685.

Mayes, S. D., \& Calhoun, S. L. (2003). Analysis of WISC-III, stanford-binet:IV, and academic achievement test scores in children with autism. Journal of Autism \& Developmental Disorders, 33(3), 329-241.

Mayes, S. D., Calhyoun, S. L., \& Crites, D. L. (2001). Does DSM-IV asperger's disorder exist? Behavioral Science, 29(3), 263-271.

Minshew, N. J., Goldstein, G., Muenz, L. R., \& Patyon, J. B. (1992). Neuropsychological functioning in non-mentally retarded 
autistic individuals. Journal of Clinical and Experimental Neuropsychology, 14, 749-761.

Rumsey, J. M., \& Hamburger, S. D. (1988). Neuropsychological findings in high-functioning men with infantile autism, residual state. Journal of Clinical and Experimental Neuropsychology, 10(2), 201-221.

Russell, J. (1997). Autism as an executive disorder. Oxford: Oxford University Press.

Ryan, J. J., \& Paolo, A. M. (2001). Exploratory factor analysis of the WAIS-III in a mixed patient sample. Archives of Clinical Neuropsychology, 16(2), 151-156.

Sattler, J. M., \& Ryan, J. J. (1999). Assessment of children, revised: WAIS III supplement. LeMesa, CA: Jerome Sattler Publishing.

Shah, A., \& Frith, U. (1993). Why do autistic individuals show superior performance on the Block Design task? Journal of Child Psychology and Psychiatry, 34, 1351-1364.

Siegel, D. J., Minshey, N. J., \& Goldstein, G. (1996). Wechsler IQ profiles in diagnosis of high-functioning autism. Journal of Autism and Developmental Disorders, 26, 389-406.
Taub, G. E. (2001). A confirmatory analysis of the wechsler adult intelligence scale-third edition: Is the Verbal/performal discrepancy justified? Practical Assessment, Research \& Evaluation, 7 (22).

Tsatsanis, K. D. (2005). Neuropsychological Characteristics in Autism and Related Conditions. In F. R. Volkmar, P. Rhea, A. Klin, \& D. Cohen (Eds.), Handbook of autism and pervasive developmental disorders. New Jersey: John Wiley \& Sons. 772798.

Vermeulen, P. (2002). Beter vroeg dan laat en beter laat dan nooit. Culemborg: Centraal boekhuis b.v.

Wechsler, D. (1997). WAIS-III Nederlandstalige bewerking, technische handleiding. Lisse: Swets \& Zeitlinger.

World Health Organization. (1993). The ICD-10 classification of mental and behavioral disorders. Diagnostic criteria for research. Geneva: WHO. 